International Journal of Mechanical Engineering Research.

ISSN 2249-0019 Volume 3, Number 1 (2013), pp. 17-30

(C) Research India Publications

https://dx.doi.org/10.37622/IJMER/3.1.2013.17-30

\title{
Basic Study of a Compact Double Acting Engine Design
}

\author{
Euich Miztani \\ JEIN Institute for Fundamental Science (JIFS) \\ VBL Kyoto University, Yoshida honmachi, sakyo-ku, Kyoto 600-8813, Japan
}

\begin{abstract}
This paper is about research of a compact double acting engine design. So far, we many engineers have thought of more powerful engines. Although Wankel engine is known actually as such an accomplished engine, its design has some problems; difficult theory by the complicated piston moving, fuel wasting, friction by thrust of the rotor, and so on. Instead, there is an engine design so to say by direct driving reciprocation without connecting rod. But the early designs in the past (, by Chrysler et al.) have problems as well because they have very complicating structure. We discuss such issues and propose a different design to solve them.
\end{abstract}

Keywords: double acting engine, direct driving reciprocation, oval piston

\section{INTRODUCTION}

Historically, Scotch-Yoke type engine could be said as the first direct driving reciprocating engine, although the engine still has connecting rod. There is the engine design by R. E. Heald [1] as shown in Figure 1. However, his design makes it complicate as a result. 


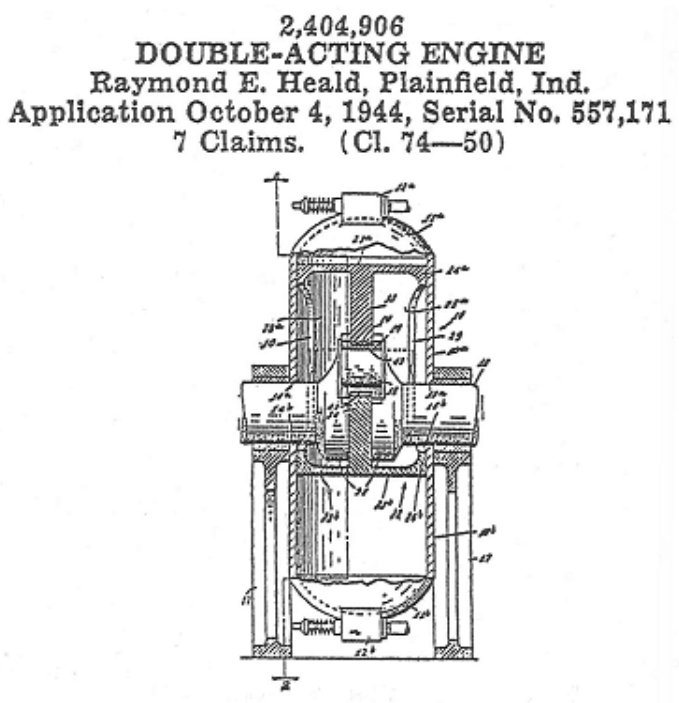

Figure 1 Heald's design

Problems of the design will be pointed out as follows;

1. Embracing the crankshaft in piston complicates the structure of doubleheaded piston.

2. According to the reason (1), it makes the piston size huge.

3. Therefore, the cylinder cannot make it compact.

4. The engine will need to divide the cylinder block into two parts to insert crankshaft on the assembly.

\section{An IMPROVED STRUCTURE}

Herein, an improved design to solve the problem is shown in Figures 1 and 2. The cross section in Figure 2. It is perspective section to embody the design. The feature is;

a. The piston (1) has two piston heads at opposite ends.

b. The piston (1) has a horizontal slot (2) for the piston pin (3) to slide in.

c. The piston pin (3) also functions as crankpin.

d. The crankshaft (4) is connected to the piston (1) through holes (5) on opposing sides of the cylinder block (6).

e. Crank arms (7) are disk and have the same diameter as holes (5) to plug them.

f. The piston (1) and cylinder (8) shapes are not cylindrical.

g. The cylinder (8) is divided two Combustion chambers by the piston (1).

Conversion of the strokes into revs is performed as follows; the piston (1) moves up and down and causes the piston pin (3) to slide right to left in the horizontal slot (2) to rev the crankshaft (4). 


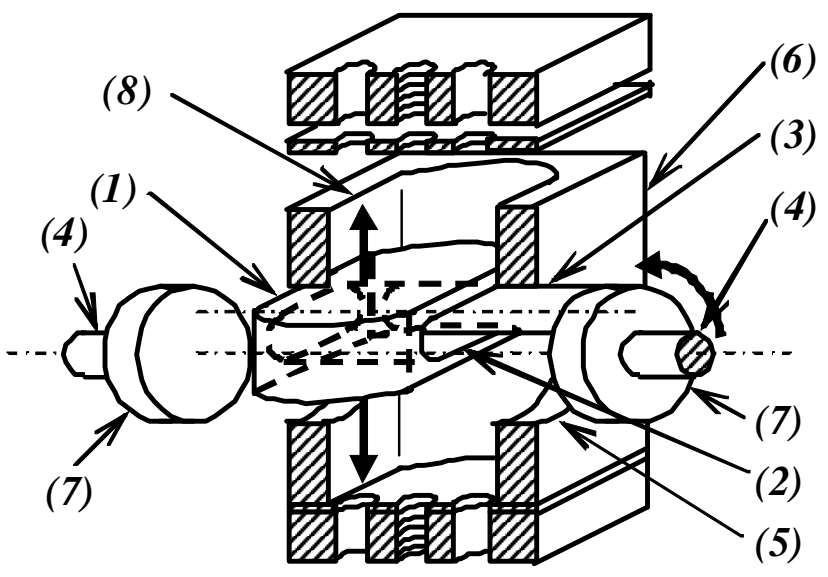

Figure 2 The Engine Design

Assembly of this engine is shown in Figure 3. It is assembled by inserting two parts of the piston (1) having bearings (9) for the slide able crank pin (3) from both of the cylinder heads, cramping the parts of pistons to connect to the crankshaft (4) which inserted from the bearings (10) to accommodate the crank arms (7) located on the center of flat parts of the cylinder (12).

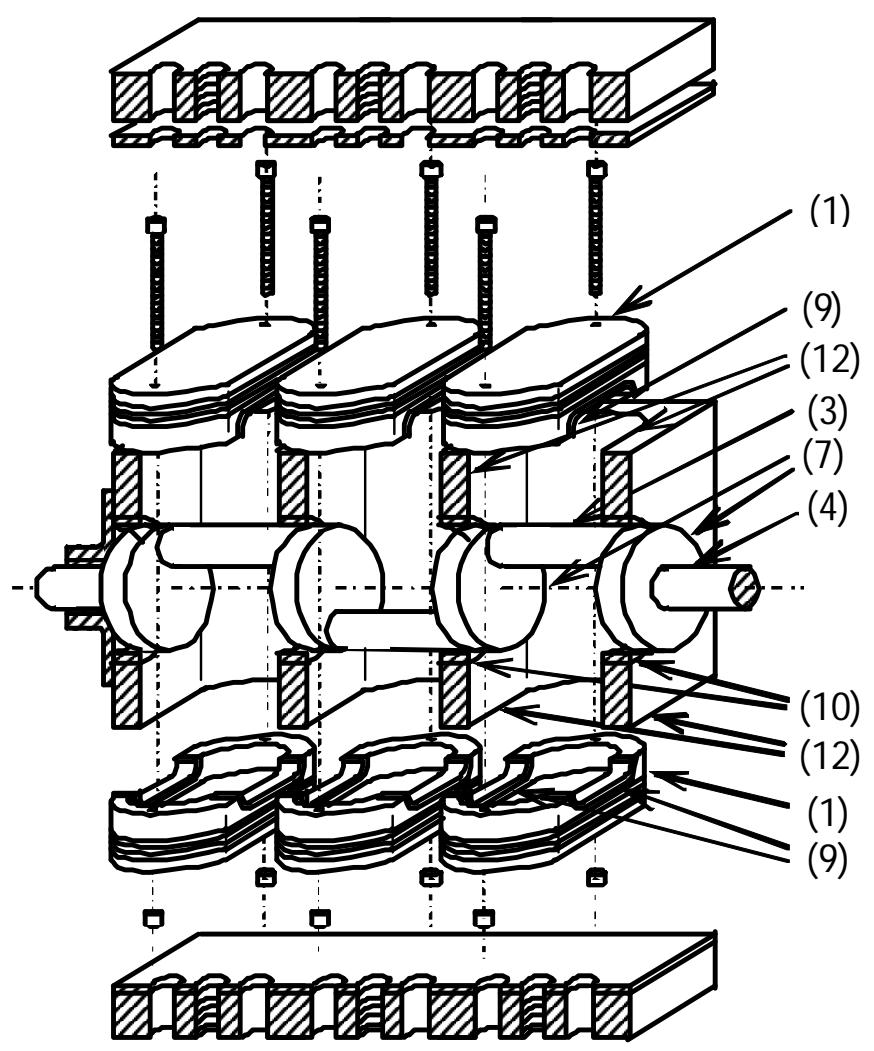

Figure 3 Assembly of the Engine 


\section{MECHANICAL PRINCIPLE}

\section{EQUATION OF STROKE-STROKE VOLUME RATIO}

The design of piston and cylinder of this engine is changeable freely by determining width of the slot side and bore of piston. It is convenient to think of the equation with bore and stroke of this double acting engine. As shown in Figure 6, the radius or bore of piston is

$$
r_{1}=\frac{L+2\left(\alpha+r_{2}\right)}{2 \sin \delta} \text { or } D=\frac{L+2\left(\alpha+r_{2}\right)}{\sin \delta}(0 \prec \delta \prec \pi / 2)
$$

where bore is $D$, stroke is $L$, and radius of the slide-able piston pin $r_{2}$.

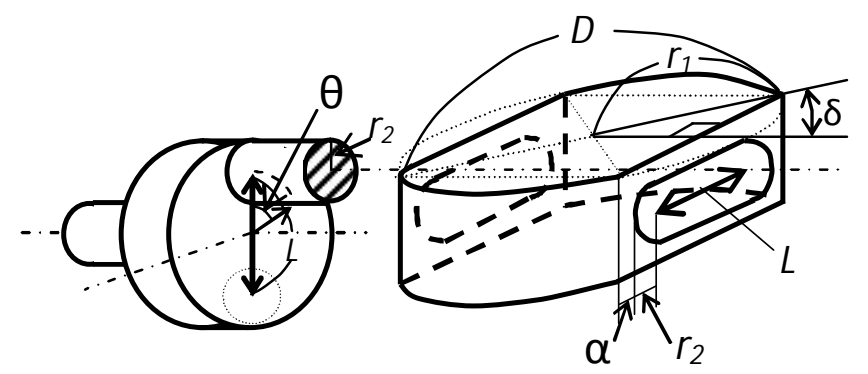

Figure 6 Measurements of the Piston and Crack Disk

The bore-stroke ratio $L / D$ is

$$
\frac{L}{D}=\frac{L}{2 r_{1}}=\frac{L}{L^{\prime} / \sin \theta}=\frac{L}{L^{\prime}} \sin \theta \prec 1
$$

The inequality (2) suggests that this engine will be preferable to be designed as square engine.

\section{PHASE, VELOCITY, ACCELERATION OF THE PISTON}

Let us think of them with the structure of engine design. As shown in Figure 7 , let the length of crank be $R$, crank angle $\theta$, location of piston as $\theta=0$ does $A$, and phase of piston $A P=z$, the phase equation is

$$
z=R(1-\cos \theta)
$$




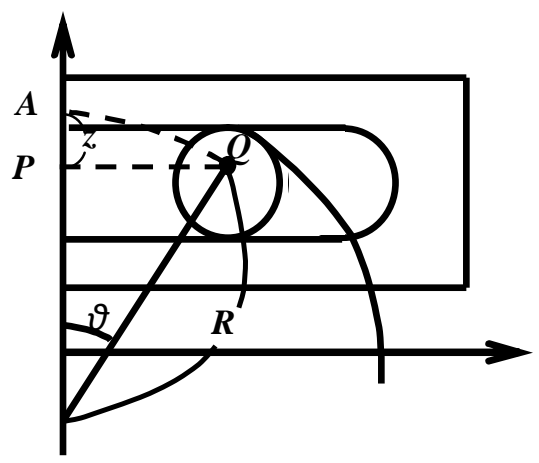

Figure 7 Piston's Location to the Stroke

The velocity of piston is

$$
v=\frac{d z}{d t}=\frac{d z}{d \theta} \frac{d \theta}{d t}=R \omega \sin \theta
$$

If $d v / d \theta=0$, then the velocity is maximum. It is at $\theta=90$ degree and 270 degree. The acceleration is

$$
\alpha=R \omega^{2} \cos \theta
$$

If $d \alpha / d t=0$, then the acceleration is maximum. It is at $\theta=0$ degree and 180 degree. Unlike Otto engine, the top speed and acceleration of piston heads are at the top (and bottom) dead center. The results are indicated by Graph 1 and Graph 2 (cf. dot-dashed curves in their graphs are Otto engine's velocity and acceleration.)

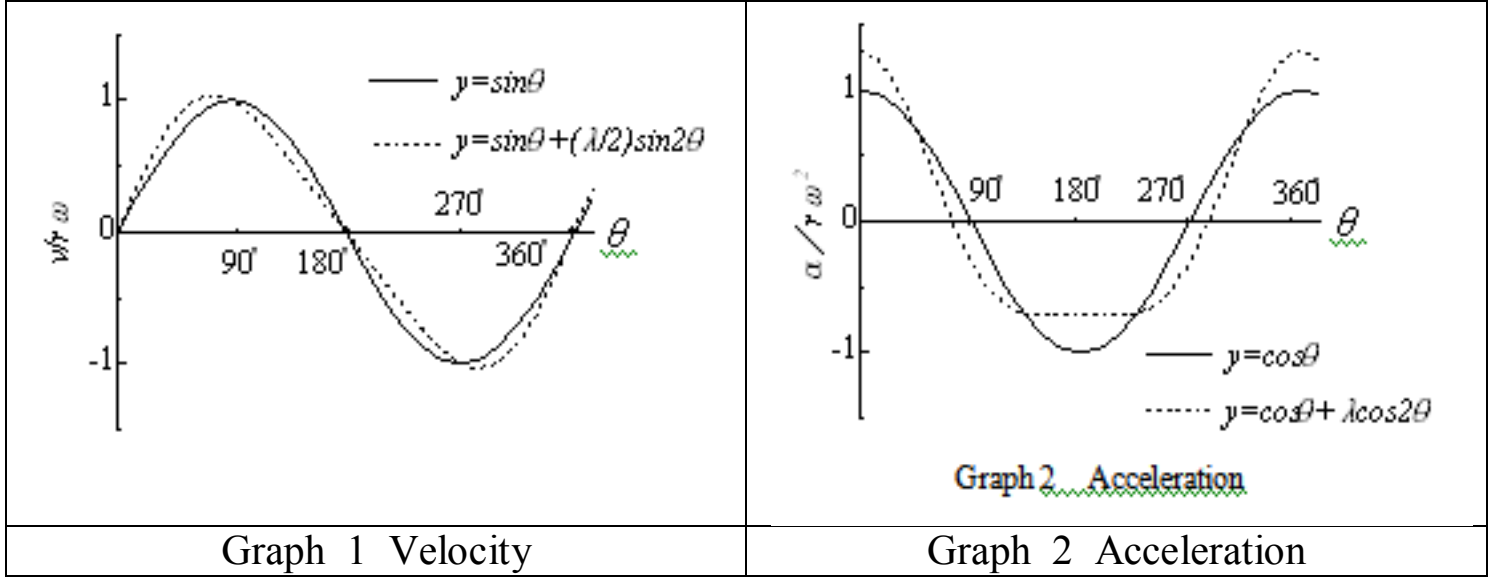

Since the cosine curve of acceleration based on this engine design is more moderate than Otto engine's, it could be easier to control Ignition Homogeneous Charge Compression Ignition (HCCI) if applying the technology for this engine design. 


\section{TORQUE}

As shown in Figure 8, let the force to piston pin by gas pressure in combustion chamber be $F$, centripetal force to the origin $O$ be $F_{r}$, and component force vertical to $F_{r}$ is $F_{t}$. Then $F_{t}$ and $F_{r}$ are

$$
F_{t}=F \sin \theta,(6) \quad F_{r}=F \cos \theta
$$

The torque $T$ is

$$
T=F_{t} \times R=F \sin \theta \times R
$$

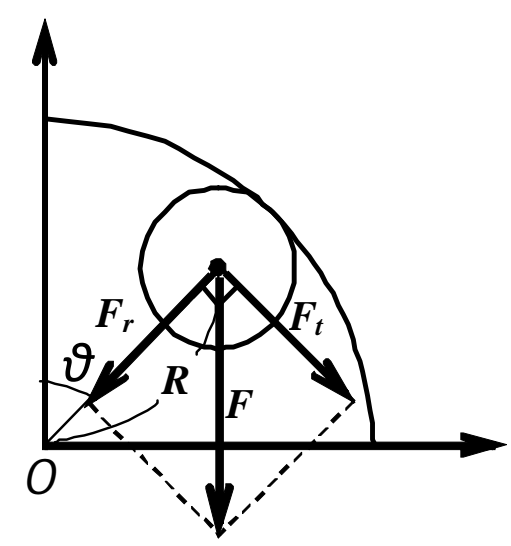

Figure 8 Force to the Piston

The graph of torque is shown in Graph 3.

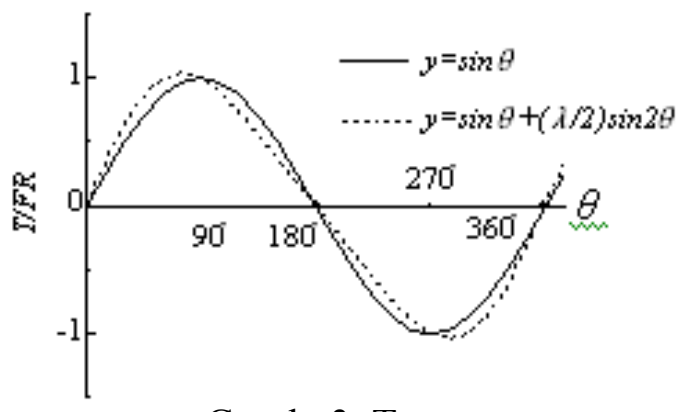

Graph 3 Torque

\section{THRUST}

As shown in Figure 9, the thrust to cylinder is related with two kinetic friction factors $\mu_{1}, \mu_{2}$. The former factor is occurred by the thrust to cylinder and the latter by the slide-able piston pin sliding in the slot. Therefore, the equation of total thrusts is

$$
F_{\text {thrust }}=\mu_{1} \mu_{2} F \sin \theta
$$


The effect by $\mu_{2}$ is considerably small because the piston pin is always sliding and unfixed. Thus, the thrust will be almost nothing. We could consider that it has almost no wear problems such by engine friction.

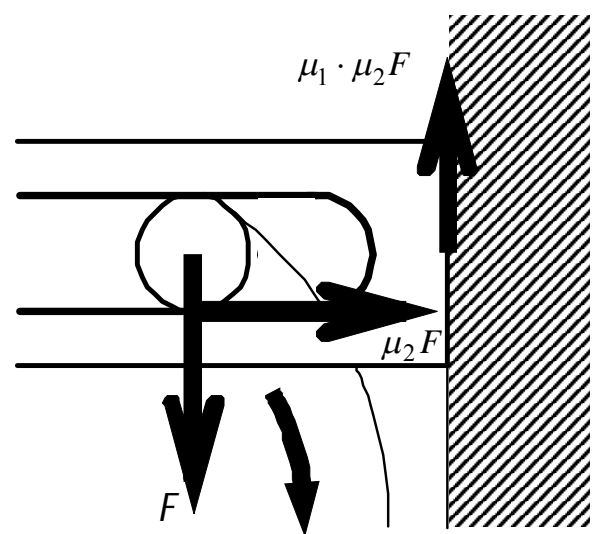

Figure 9 Thrust and Frictions

\section{BALANCE}

Setting this double acting engine stroke horizontally, the engine vibration will be considerably reduced so that the balance will be unnecessary significant. On the other hand, it may have a different function in this engine design. It is about leveling the non fixed piston by the piston pin. As shown in Figure 10, the equation of balancing is

$$
-m_{p} R^{2}+m_{b p} R_{b}^{2}=0
$$

If $m_{p} R^{2}=m_{b p} R_{b}^{2}$, then it keeps the balance of moments. Similarly, the equation of inertia forces for the balance is

$$
m_{c p} R^{2}=m_{b q} R_{b}^{2}
$$

where $m_{c p}$ and $m_{b q}$ are inertial masses of the piston and balance. Considering over balancing, the total mass of balance $m_{b}$ is

$$
m_{b}=k m_{b p}+m_{b q}
$$

where $k$ is parameter for adjustment of balance.

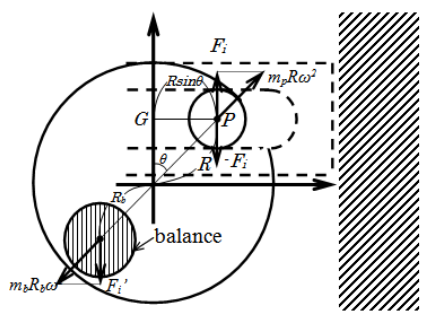

Figure 10 Balance 


\section{THERMODYNAMITCS}

Otto cycle is basically based on a single combustion chamber. However, in this case of double acting engine, some effects by mutual gas pressures from both two combustion chambers are considered as shown in Figure 11. In this chapter, we think of the thermodynamics of double acting engine and characterization of the theoretical cycle within the first law of thermodynamics.

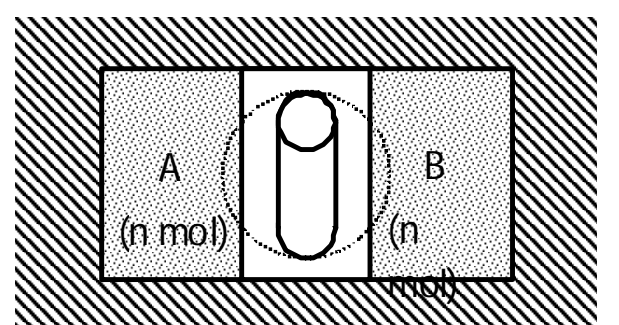

Figure 11 Ideal Gases on both Chambers

\section{THERMODYNAMICS BY OTTO CYCLES}

In general, the low of thermal efficiency of Otto cycle is

$$
\eta=\frac{Q_{1}-Q_{2}}{Q_{1}}
$$

In fact, the result of Otto cycle by two chambers on both sides of the double headed piston is theoretically the same as the original equation (13) under special conditions(, refer to ANNEX.) Therefore, we could conclude it as follows;

1. The thermal efficiency by two chambers is all the same as conventional one under conditions $P_{b} \geq P_{b}^{\prime}$ and $P_{d}^{\prime} \geq P_{d}$, where $P_{b}$ and $P_{d}^{\prime}$ are the highest pressures in the process of compression in chambers $\mathrm{A}$ and $\mathrm{B}$, $P^{\prime}{ }_{b}$ and $P_{d}$ the lowest ones in the process of exhaust.

2. For the reasons listed above, this double acting engine will be advantageous on the power efficiency in terms of reduction of mechanical energy loss by simpler structure than two trains of Otto engines.

3. If $P_{b} \geq P_{b}^{\prime}$ and $P_{d}^{\prime} \geq P_{d}$, then these conditions will offer the most ideal thermal efficiency because of less consideration of flywheel to prevent reversing of engine.

\section{CONCLUSIONS}

This engine design has double-powered by combustions on both sides, moreover simple and compact. It seems to be advantageous in many aspects. On the other hand, there will be unknown problems for practical use. Therefore, it will need research experiments for the next stage. 


\section{NOMENCULTURE}

Direct Driving Reciprocation (DDR)

Ignition Homogeneous Charge Compression Ignition (HCCI)

Scotch-Yoke type engine

Double acting engine

Otto engine

Wankel engine

\section{REFERENCES}

[1] Raymond E. Heald, Application, October 4, 1944, Serial No. 557,171 (Document No. 2,404,906), U.S. Patent Office

[2] John B. Platner, assignor to Chrysler Corporation, Application: March 24, 1948, Serial No. 16,688 (Document No. 2,562,639), U.S. Patent Office

[3] Arthur J. Slemmons, assignor to Chrysler Corporation, Application: March 10, 1949, Serial No. 80,729 (Document No. 2,588,666), U.S. Patent Office

[4] Alexander G. Herreshoff, assignor to Chrysler Corporation, Application: March 24, 1948, Serial No. 16,801(Document No. 2,613,651), U.S. Patent Office Furthermore, as the predecessor of oval piston engine, see also: http://world.honda.com/history/challenge/1979pistonengine/index.html

\section{AKNOWLEDGEMENTS}

Acknowledgements to Prof. Masako Bando the director of my research institute JIFS and also a former chair of Physical Society of Japan, and Prof. Takuya Matsuda the vice director and also Professor Emeritus of Kobe University for their supports.

\section{ANNEX}

\section{EQUATION OF STROKE-STROKE VOLUME RATIO}

As shown in Figure 12, the area of piston head $\mathrm{S}$ (; integration of half hemicycle) is

$$
S=4 \int_{0}^{r_{1} \cos \delta} \sqrt{r_{1}^{2}-x^{2}} d x
$$

Substituting $x=r_{1} \sin \varphi$ and $d x / d \varphi=r_{1} \cos \varphi$ in the equation above,

$$
S=4 r_{1}^{2} \int_{0}^{(\pi / 2)-\delta} \cos ^{2} \varphi d \varphi
$$




$$
\begin{aligned}
& =4 r_{1}^{2} \int_{0}^{(\pi / 2)-\delta} \frac{1+\cos 2 \varphi}{2} d \varphi \\
& =r_{1}^{2}(\pi-2 \delta+\sin 2 \delta)
\end{aligned}
$$

Substituting the equation (1) in the eq. (14),

$$
S=\left(\frac{L+2\left(\alpha+r_{2}\right)}{2 \sin \delta}\right)^{2}(\pi-2 \delta+\sin 2 \delta)
$$

Multiplying the eq. (15) by stroke $L$, the stroke volume $V$ is

$$
\begin{aligned}
& V=L\left(\frac{L^{\prime}}{2 \sin \delta}\right)^{2}(\pi-2 \delta+\sin 2 \delta) \\
& =L\left(\frac{D}{2}\right)^{2}(\pi-2 \delta+\sin 2 \delta)
\end{aligned}
$$

where $L^{\prime}=L+2\left(\alpha+r_{2}\right)$.

From the equation (16), the stroke $L$ is

$$
L=\sqrt{\left(\frac{4}{(\pi-2 \delta+\sin 2 \delta) D^{2}}\right) V}
$$

The equation of stroke-stroke volume ratio is defined as

$$
L^{2}=\left(\frac{4}{\pi-2 \delta+\sin 2 \delta}\right) D^{-2} V
$$

If substituting $\delta=\frac{\pi}{4}$,

$$
L^{2}=\left(\frac{8}{\pi+2}\right) D^{-2} V
$$

Additionally, considering the stroke volume by the crank angle $\theta$, the upper combustion chamber's stroke volume is

$$
V_{\text {upper }}=\frac{L}{2}(1-\cos \theta)\left(\frac{D}{2}\right)^{2}(\pi-2 \delta+\sin 2 \delta)
$$

At the same time, the lower chamber's is

$$
V_{\text {lower }}=\frac{L}{2}(1+\cos \theta)\left(\frac{D}{2}\right)^{2}(\pi-2 \delta+\sin 2 \delta)
$$


These equations indicate each stroke volume by transition of the crank angle.

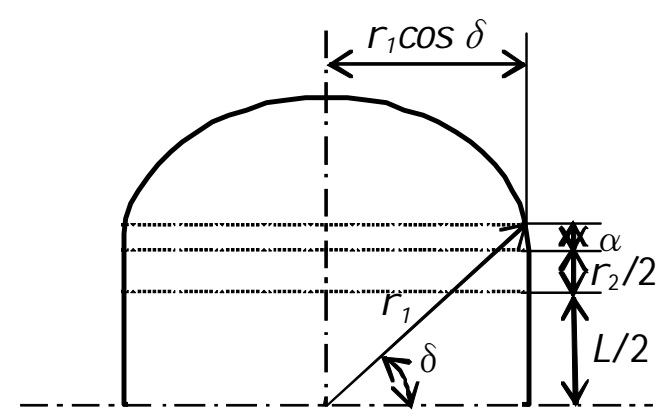

Figure 12 Measurements of the Piston

\section{BALANCE}

As shown in Figure 10, the force of inertia or concentrated load to piston by stroke $F_{i}$ is

$$
F_{i}=-m_{p} R \omega^{2} \cos \theta
$$

$m_{p}$ denotes weight of the piston, $\omega$ angular velocity. It occurs a couple to the piston because the center of gravity of piston does not always correspond to the slide-able piston pin's. It is the reason of the piston's unbalance in reciprocating. The moment of a couple $M_{1}$ around the origin $O$ is

$$
\begin{aligned}
& M_{1}=-F_{i} \times R \sin \theta \\
& =-m_{p} R^{2} \omega^{2} \sin \theta \cos \theta
\end{aligned}
$$

This engine design needs a balance for the moment therefore. The moment by balance is

$$
\begin{aligned}
& M_{2}=F_{i}{ }^{\prime} \times R_{b} \sin \theta \\
& =m_{b p} R_{b}{ }^{2} \omega^{2} \sin \theta \cos \theta
\end{aligned}
$$

$F_{i}$ ' denotes the component force vertical to centrifugal force by balance, $m_{b}$ weight of balance, and $R_{b}$ distance between the origin and center of gravity of the balance. Since the equation (23) should be equal to the equation (24),

$$
M_{1}+M_{2}=0
$$

From these equations (23) and (24), the equation (25) is

$$
-m_{p} R^{2}+m_{b p} R_{b}^{2}=0
$$




\section{THERMODYNAMICS BY THE OTTO CYCLES}

Otto cycle is determined by heat input and output in isobaric changes in general. Now, two Otto cycle indicators diagrams are shown in Graph 4. The pressures, volumes, and temperatures of each point $a, b, c, d$ and $a^{\prime}, b^{\prime}, c^{\prime}, d^{\prime}$ on the diagrams $A, B$ are related with,

$$
\begin{array}{ll}
\left(P_{a}, V_{a}, T_{a}\right)=\left(P_{c}, V_{c}{ }^{\prime}, T_{c}{ }^{\prime}\right), & \left(P_{b}, V_{b}, T_{b}\right)=\left(P_{d}, V_{d}, T_{d}{ }^{\prime}\right), \\
\left(P_{c}, V_{c}, T_{c}\right)=\left(P_{a}, V_{a}, T_{a}\right), & \left(P_{d}, V_{d}, T_{d}\right)=\left(P_{b}, V_{b}, T_{b}{ }^{\prime}\right) .
\end{array}
$$

The order of passages in the chamber $A$ is $a \rightarrow b \rightarrow c \rightarrow d$, at the same time the chamber $B$ is $a^{\prime} \rightarrow b^{\prime} \rightarrow c^{\prime} \rightarrow d^{\prime}$. Additionally, we set conditions $P_{b} \geq P_{b}^{\prime}$ and $P_{d}^{\prime} \geq P_{d}$ not to reverse the orders of passages in the reciprocation. Since the total volume of chambers $A$ and $B$ is constant,

$$
V_{a}+V_{a}{ }^{\prime}=V_{b}+V_{b}{ }^{\prime}=V_{c}+V_{c}{ }^{\prime}=V_{d}+V_{d} \text {. }
$$

Processes $a-b, c^{\prime}-d^{\prime}$, and $c-d, a^{\prime}-b^{\prime}$ are adiabatic change, and Process $b-c$, $d^{\prime}-a^{\prime}, d-a$, and $b^{\prime}-c^{\prime}$ are isochoric change.

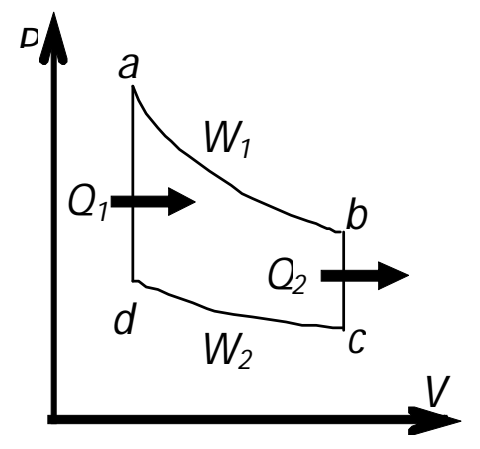

Diagram A

(Chamber A)

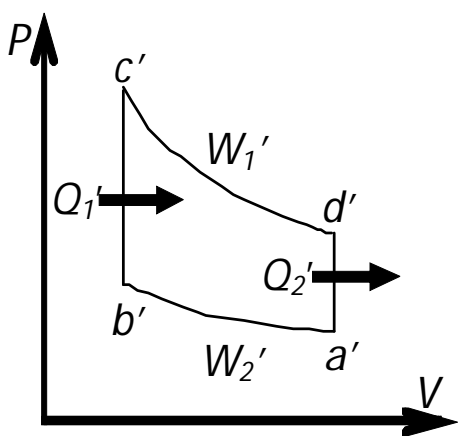

Diagram B (Chamber B)

Graph 4

In the chamber $A$, the power by process $a-b$ (without considering effect by the chamber $B$ ) $W_{l}$ is generally,

$$
W_{1}=Q_{1}=\int_{a}^{b} P_{\text {lint }} d V=K_{a b} \int_{a}^{b} \frac{d V}{V^{\gamma}}=\frac{K_{a b}}{\gamma-1}\left(V_{a}^{-\gamma+1}-V_{b}^{-\gamma+1}\right)
$$

where $P_{i n t}$ is gas pressure in the chamber A.

Since $K_{a b}=p_{a} V_{a}^{\gamma}=p_{b} V_{b}^{\gamma}$, the equation (27) is

$$
W_{1}=\frac{1}{\gamma-1}\left(p_{a} V_{a}-p_{b} V_{b}\right)=\frac{n R}{\gamma-1}\left(T_{a}-T_{b}\right)
$$


On the other hand, the process $a^{\prime}-b^{\prime}$ in chamber $B$ is adiabatic compression at the same time with the process $a-b$. This power $W_{2}{ }^{\prime}$ is

$$
W_{2}^{\prime}=Q_{2}^{\prime}=Q_{2}=\int_{c}^{d} P_{2 e x t} d V=\int_{c}^{d} P_{2 e x t} d V=K_{c d}^{\prime} \int_{c}^{l} \frac{d V}{V^{\gamma}}=\frac{K_{c d}}{\gamma-1}\left(V_{d}^{-\gamma+1}-V_{c}^{-\gamma+1}\right)
$$

where $P_{\text {ext }}$ is gas pressure in the chamber B.

Since $K_{c d}^{\prime}=K_{c d}=p_{c} V_{c}^{\gamma}=p_{d} V_{d}^{\gamma}$,

$$
W_{2}{ }^{\prime}=\frac{1}{\gamma-1}\left(p_{c} V_{c}-p_{d} V_{d}\right)=\frac{n R}{\gamma-1}\left(T_{d}-T_{c}\right)
$$

As the result, the power $W_{\alpha}$ by process $a-b$ and $a^{\prime}-b^{\prime}$ is

$$
\begin{aligned}
& W_{\alpha}=W_{1}-W_{2}^{\prime}=Q_{\alpha}=\int_{a}^{b} P_{\alpha} d V=\int_{a}^{b}\left(P_{\text {lint }}-P_{2 \text { int }}^{\prime}\right) d V=\int_{a}^{b} P_{\text {lint }} d V-\int_{c}^{d} P_{\text {2ext }} d V \\
& =\frac{n R}{\gamma-1}\left[\left(T_{a}-T_{b}\right)-\left(T_{d}-T_{c}\right)\right]
\end{aligned}
$$

Similarly, the power $W_{\beta}$ by process $c-d$ and $c^{\prime}-d$ is

$$
W_{\beta}=\frac{n R}{\gamma-1}\left[\left(T_{d}-T_{c}\right)-\left(T_{a}-T_{b}\right)\right]
$$

The total work output is

$$
W_{\alpha}-W_{\beta}=\frac{2 n R}{\gamma-1}\left[\left(T_{a}-T_{b}\right)-\left(T_{d}-T_{c}\right)\right]
$$

Arranging members in the brace on the right side of equation (33),

$$
\begin{aligned}
& W_{\alpha}-W_{\beta}=\frac{2 n R}{\gamma-1}\left(T_{a}-T_{d}+T_{c}-T_{b}\right) \\
& =\frac{2 n R}{\gamma-1}\left[\left(T_{a}-T_{d}\right)-\left(T_{b}-T_{c}\right)\right]
\end{aligned}
$$

The thermal efficiency of this cycle $\eta$ is

$$
\eta=\frac{W_{\alpha}-W_{\beta}}{2 Q_{1}}=\frac{n R}{Q_{1}(\gamma-1)}\left[\left(T_{a}-T_{d}\right)-\left(T_{b}-T_{c}\right)\right]
$$

since $Q_{1}=C_{V}\left(T_{a}-T_{d}\right), \mathrm{Q}_{2}=C_{V}\left(T_{b}-T_{c}\right), C_{V}=\frac{3}{2} n R$, and $\gamma \equiv C_{P} / C_{V}=\frac{5}{2} n R / \frac{3}{2} n R=\frac{5}{3}, \quad$ the equation (35) is 


$$
\begin{aligned}
& \eta=\frac{\frac{2}{3} C_{V}\left[\left(T_{a}-T_{d}\right)-\left(T_{b}-T_{c}\right)\right]}{\frac{2}{3} C_{V}\left(T_{a}-T_{d}\right)} \\
& =1-\frac{T_{b}-T_{c}}{T_{a}-T_{d}}\left(=\frac{Q_{1}-Q_{2}}{Q_{1}}\right)
\end{aligned}
$$

The equation (36) equals to conventional efficiency as a result. 